dingt auf ihre familiären Netzwerke verlassen können. Auf der anderen Seite gibt es gerade in Juba eine nicht zu unterschätzende Zahl an Young Professionals, die sich zur Förderung ihrer beruflichen Karriere für eine späte Heirat und weniger Kinder entscheiden und sich somit der geschlechtlichen Verwertungsökonomie entziehen. Diese Frauen sind keineswegs nur im Sektor der Internationalen Organisationen und der professionellen Zivilgesellschaft $\mathrm{zu}$ finden. Doch ihre hybride Situation schlägt nicht selten auf ihre persönliche Situation durch: die soziale Erwartungshaltung, zu heiraten und eine Familie zu gründen, ist nach wie vor dominant. Die Akzeptanz von alternativen Lebensmodellen, abseits offizieller Lippenbekenntnisse, ist dahingegen nicht sehr ausgeprägt. Änderungen sind jedoch spürbar. Auch wenn eine südsudanesische Frauenbewegung aus historischen Gründen schwere Bedingungen vorfinden würde (Edward, 2019), nimmt die Zahl an Frauen, die strukturelle Veränderungen aktiv und organisiert vorantreiben, kontinuierlich zu.

\title{
A good place to make money
}

Es gehört zu den Besonderheiten der südsudanesischen Wirtschaftskrise, dass die Attraktivität der größeren Städte für internationale Arbeitskräfte ungebrochen ist. Sudanesische Händler in Bentiu oder ugandische Arbeiter in Yei sind leicht $\mathrm{zu}$ finden. Juba ist ohnehin nicht nur ein Schmelztiegel von Arbeits- und Glückssuchenden aus den umliegenden Ländern. Es ist ein "good place to make money«, der auch eine relevante Zahl an libanesischen und chinesischen Geschäftsleuten anzieht.

Die Boomzeit der CPA-Periode Ende der 20ooer-Jahre, als Tausende junge Ugander:innen und Kenianer:innen nach Juba zogen, um dort schnelles Geld zu machen oder doch zumindest einen annehmbar bezahlten Job zu finden, war eine in vieler Hinsicht beeindruckende Periode. Freunde in Khartum fragten mich nach den Perspektiven, auf Kredit Prefab-Einheiten zu kaufen und sie in Juba für ein weiteres »Camp« zu nutzen. Internationale Hilfskräfte zahlten zu jener Zeit mehrere tausend US-Dollar, um in solchen Prefabs zu wohnen. Pro Monat. In Juba war es zu jener Zeit so gut wie unmöglich, einen Boda Boda-Fahrer zu finden, der nicht erst kürzlich aus Uganda in die Stadt gekommen war, um mit seinem Motorrad mit hohem körperlichem Risiko, aber vergleichsweise einfach Geld zu verdienen. Nicht nur einmal scheiterten meine damaligen Bemühungen, eine Destination in der Stadt anzusteuern, an einem mir unbekannten Ort ohne Straßennamen. Ohne verfügbare Karte 
und ohne Ortskenntnis eine beiderseitige Herausforderung. "You show me the way.«»How should I know?«

Spätestens mit dem Ende des kurzlebigen Ölbooms Anfang der 2010erJahre bricht der überbordende Optimismus. Die Karawane der ökonomischen Glücksritter zieht weiter in das 300 Kilometer südlich gelegene Gulu in Norduganda, das nach Ende des bewaffneten Konfliktes mit der LRA einen rapiden ökonomischen Aufschwung zu einer regionalen Handelsmetropole durchlebt. Im Südsudan wiederum führt der Bürgerkrieg zu einem markanten ökonomischen Einbruch. Das GDP pro Kopf fällt von fast 1.800 US-Dollar im Jahr 2013 - einem mit Kenia vergleichbaren Wert - auf ganze 200 US-Dollar im Jahr 2017. Die Regierung reagiert zunächst mit nationalistischen Reflexen und versucht im September 2014, ausländische Arbeitskräfte vom ohnehin kleinen regulären Arbeitsmarkt per Gesetz auszuschließen. Nach massivem Druck Ugandas und der anzunehmenden Einsicht in die beschränkte Wirksamkeit einer solchen Maßnahme wird davon wieder Abstand genommen. ${ }^{4}$

Was eingeführt und bis heute aufrechterhalten wird, sind jedoch schmerzhaft teure Bescheinigungen, die eine Arbeitserlaubnis bestätigen: Work Permits. Eine solche Bescheinigung haben alle ausländischen Arbeitskräfte $\mathrm{zu}$ erwerben und auf Nachfrage vorzuweisen. Nach einem kurzfristigen Anstieg auf bis $\mathrm{zu} 10.000$ US-Dollar für einige Positionen haben sich die Gebühren Ende der 2010er-Jahre auf 1.000 bis 2.000 USDollar eingependelt. Dies sind dennoch astronomische Posten für die vielen kleinen Shops in den Märkten, die oft Personal aus den Nachbarländern beschäftigen. Somit werden diese für viele nicht leistbaren Gebühren zu einem Einfallstor für informelle Abgaben an (potenziell) kontrollierende Staatsbedienstete.

Mittlerweile hat die ökonomische Krise voll durchgeschlagen. Staatsbedienstete werden ab Mitte des Jahres 2019 zum Teil über mehrere Monate nicht bezahlt, was wiederum auf den Dienstleistungssektor durchschlägt. Verspätete oder ausgefallene Zahlungen von Löhnen, die in ihrer Höhe ohnehin mehr schlecht als recht zum Überleben reichen, sind in allen Teilen des Landes mit einer etablierten Lohnarbeitsstruktur gang und gäbe. Es ist zutiefst erstaunlich, wie viele trotz der fehlenden Löhne weiterarbeiten, oft eingebettet in die soziale Struktur ihrer Betriebe und verstrickt in ein weitläufiges Netz an privater Geldleihe. Das Niveau der Krise lässt sich an verschiedenen Vergleichen des Wertes des SSP festmachen. Begeben im Juli 2011 mit 
der fixierten Rate von 2,96 SSP zum US-Dollar, der gleichen Rate wie das sudanesische Pfund, hält sich der Wert über lange Zeit relativ stabil. Ende des Jahres 2015 entscheidet sich die südsudanesische Regierung zu einer kurzfristigen Freigabe des Wechselkurses. ${ }^{5}$ Offizieller Markt und Schwarzmarkt nähern sich 20 SSP zu einem US-Dollar an.

Bis Mitte des Jahres 2017 ist die Inflation stark, aber sie verläuft zumindest graduell. Der Unterschied zwischen Bank- und Straßen-Markt bewegt sich in überschaubaren Grenzen: 130 SSP per US-Dollar im Juni 2017 am offiziellen Markt, 150 am informellen. Ab da wird es rasant. Bereits ein Jahr später ist der Schwarzmarkt-Wert des SSP halbiert, während der offizielle Wechselkurs beibehalten wird. Bis zum Januar 2021 bleibt der offizielle Wechselkurs bei den 130 SSP per US-Dollar fixiert, während die Währung im Verlauf des Jahres 2020 im realen Wert eine hyperinflationäre Entwicklung erlebt: Ende des Jahres kostet ein US-Dollar am Schwarzmarkt 600 SSP. Zum Vergleich: Das mit gleichem Wert gestartete sudanesische Pfund steht am Ende des Jahres 2020 am parallelen Markt bei 250 per US-Dollar. Er ist also mehr als doppelt so stark, wobei selbst dieser Wert Ausdruck einer ökonomischen Krise im Sudan ist, die letztlich in einer sozialen Revolution resultiert.

Diese Zahlen repräsentieren konkrete Lebensrealitäten. Viele der auf den Märkten erhältlichen Produkte, selbst landwirtschaftliche, sind aus Nachbarländern importiert. Dies ist nicht zuletzt eine Kriegsfolge, und sie macht die sozioökonomischen Umstände auch in peripheren Regionen spürbar vom USDollar-Wechselkurs abhängig. In SSP ausgezahlte Löhne verlieren beständig an Kaufkraft. Importierte Güter sind teuer. Eine kleine Flasche Coca-Cola bewegt sich etwa im PoC/IDP Camp in Bentiu, wechselkursabhängig, bei umgerechnet einem US-Dollar. In einem Vertriebenencamp ist dies eine unerschwingliche Summe. Kosten für Kommunikation, nach dem Essen für viele das zweitwichtigste Gut, sind ebenso massiv. Smartphones kosten in USDollar-Preisen mehr als in Europa. Während sich derzeit die Kosten für nationale Mobiltelefonie noch in überschaubaren Größen bewegen - ein längeres Telefonat innerhalb des Landes ist für umgerechnet 50 US-Cents jedenfalls machbar - haben die Preise für mobile Daten mittlerweile derart angezogen, dass sie diejenigen in Europa bei weitem übertreffen.

Der Markt schließt aus, der Markt treibt, der Markt ermöglicht. Für jene nicht wenigen Menschen in vorwiegend abgeschiedenen Gemeinschaften, 
die sich am Geldmarkt bislang nicht beteiligen, ist ein Markteintritt derzeit so gut wie unmöglich. Viele in den Städten wiederum schlittern in Lethargie und versuchen, in ihren Familienverbänden ein von Tag zu Tag ausgerichtetes, kostenvermeidendes Leben zu organisieren. Wieder andere zwingt die Geldknappheit in kreative oder, in zunehmendem Maße, gewaltsame Formen der Geldbeschaffung. Hungrige Soldat:innen an nächtlichen Checkpoints, die höflich um einen Beitrag zu ihrem Abendessen bitten, oder wirsche Straßenpolizist:innen, die verzweifelt nach irgendeinem Vergehen Ausschau halten, um ein Zubrot zu realisieren, sind für viele lästige, aber vorhersehbare Probleme. Die rasant zunehmende Kriminalität in nahezu allen größeren Städten und sich professionalisierende Praktiken des Straßenraubs auf Landstraßen sind schwieriger zu handhaben und potenziell lebensbedrohlich.

Die Krux einer nur in Fragmenten existierenden Staatlichkeit ist, dass Revolutionen aus strukturellen Gründen, wenn nicht ganz ausgeschlossen, so doch höchst unwahrscheinlich sind. Die Regierung kann sich in einer derartigen Konstellation gar nicht zu einem uniformen Feindbild entwickeln, was ihr erstaunlicherweise bewusst sein dürfte. Im Unterschied zum Sudan, wo Omar al-Bashir im letzten Jahrzehnt seiner Amtszeit mehrere Brotunruhen erleben musste und niederschlagen ließ, ist der Südsudan trotz einer ökonomisch viel disparateren Situation weit davon entfernt. Die Marginalisierung greift.

Zugleich bietet der Markt überraschende Möglichkeiten. Ist Startkapital vorhanden, entwickeln sich bemerkenswerte Unternehmungen und Initiativen. Die Herausforderung sind naturgemäß die Barrieren, die einen Markteintritt für all jene verhindern, die nicht auf das notwendige Kleingeld oder ein gutes Beziehungsnetzwerk zurückgreifen können (zu den Folgen solcher Eintrittsbarrieren siehe Acemoglu und Robinson, 2012). Praktisch alle meine Bekannten, die sich unternehmerisch betätigen, verfügen entweder über ein im Ausland verdientes oder familiär erworbenes Grundkapital, dessen Höhe mitunter erstaunliche Ausmaße annehmen kann. Speziell Juba ist ein Platz für Investitionen, eben der zitierte "good place to make money«. »Wir leben hier unseren Traum«, sagen zwei libanesische Freunde, die in verschiedenen Bereichen der Immobilienentwicklung tätig sind. Die Bedingungen im ebenso von einer disruptiven ökonomischen Krise erschütterten Libanon wären nicht annähernd so günstig.

Das ist kein schlechtes Zeichen. Die ökonomische Krise produziert nicht nur Ausschluss und Marginalisierung und perpetuiert Vulnerabilität. Es gibt wirtschaftliche Dynamik und es gibt jene, die risikobereit und willens genug 
sind, diese Dynamik zu entdecken und auszunützen. Es wäre naiv anzunehmen, dass diese Prozesse kurz- oder selbst mittelfristig die Lebensbedingungen eines relevanten Teils der Menschen im Land substanziell verbessern können. Aber es wäre der sprichwörtliche Verweis auf ein halb leeres Glas, diese Prozesse als irrelevant abzutun. 
